\title{
Correlation Between Maternal Periodontal Status And Incidence of Preterm Low Birth weight Infants
}

\author{
Dr. Shashikanth Hegde ${ }^{1}$, Dr. Shalini Grover. ${ }^{2}$, Dr. Rajesh Kashyap ${ }^{3}$, \\ Dr.Arun Kumar M.S $S^{4}$, Dr.Julia.P Jacob ${ }^{5}$ \\ ${ }^{1}$ Professor \& Head Of Dept Of Periodontics, Yenepoya Dental College, Mangalore. \\ ${ }^{2}$ Private Practitioner, Shivram Dental Clinic ,Derawal Nagar,Delhi \\ ${ }^{3,4}$ Professor, Department Of Periodontics, Yenepoya Dental College, Mangalore \\ ${ }^{5}$ Post Graduate, Department Of Periodontics, Yenepoya Dental College, Mangalore
}

\begin{abstract}
Background and Objectives: Low birth weight (LBW), defined as birth weight less than 2.500 kilograms (kg), continues to be a significant public health issue in both developed and developing countries. This obstetric complication is usually a result of pre-term labor, in which case it is referred to as pre-term delivery (less than 37 weeks) of low birth weight infants (PLBW). Various factors have been associated with the delivery of PLBW infants which includes maternal risk factors such as infection, age, height, weight, SES, ethnicity, smoking, nutritional status, and stress. Periodontal diseases are a group of infectious diseases resulting in inflammation of gingival and periodontal tissues. These infections may constitute remote maternal infections that may adversely influence birth outcome. The studies shows an association between the existence of periodontal diseases and PTLBW. The objectives determine the association between periodontal status of the pregnant women and $P T B, L B W$ and $P L B W$.

Method: 200 postpartum women were included in this study. This study population was grouped into Group I(Term normal weight infants, TNBW)and Group II(cases) further divided into Subgroup A (Preterm normal weight, PNBW), Subgroup B: (Term low birth weight, TLBW) and Subgroup C: (Preterm low birth weight, $P T L B W)$. The status of both the groups was assessed within 24 hrs of delivery by using GI and CPI.

Results: This study concluded that the severity of disease appeared to be worsened as the maternal age increased. An increase in the GI and PI score was observed in cases compared to controls.

Interpretation and conclusion: There is statistically significant association between the periodontal status of the pregnant women and PTB, $L B W$ and PLBW
\end{abstract}

Keywords: Birth weight; periodontal disease; Preterm low birth weight

\section{Introduction}

Oral health has been associated with pregnancy for generations. The famous saying "a (loss of a) tooth for every child" is still heard in many parts of the world. We all are aware of the effect of pregnancy on the oral health status of the pregnant woman. Miller originally published his "focal infection theory" in 1981 suggesting that "microorganisms or their waste products obtain entrance to parts of the body adjacent or remote from the mouth". The focal infection theory was not revisited for the next 50 years. The hypothesis that infection remote from the fetal placental unit may influence PLBW has led to an increased awareness of the potential role of chronic bacterial infections elsewhere in the body. Periodontal disease is associated with a chronic Gramnegative infection' of the periodontal tissues which results in long-term local elevation of pro-inflammatory prostaglandins and cytokines and an increase in the systemic levels of some of these inflammatory mediators. Hence, periodontal disease has a potential to influence PLBW through an indirect mechanism involving inflammatory mediators or a direct bacterial assault on the amnion.[2]

Women with asymptomatic bacteriuria or abnormal colonization by pathogenic micro-organisms are twice as likely to deliver preterm infants as a result of spontaneous preterm labor, pre-labor rupture of membranes, and chorioamnionitis. The etiological role of maternal infection, either in the genital tract or elsewhere, on preterm delivery remains controversial but may be an indirect effect as a consequence of the production of increased levels of inflammatory mediators which shorten gestational age. It is also plausible that micro-organisms may gain direct access to the amniotic fluid and fetus in several ways: ascending via the vagina through the cervix into the choriodecidual sac during pregnancy, via the endometrium which may be chronically infected prior to pregnancy, or alternatively through a hematogenous route.In view of the implication of periodontal infection as one of the risk factor in PTLBW, this study was sought to determine whether the prevalence of periodontal infection could be associated with preterm low birth weight controlling all the known obstetric risk factors and potential covariates. 


\section{Materials And Methods}

The patients for this study were selected from Department of Obstetrics and Gynecology, Fr. Muller's Medical College Hospital, Mangalore. The consent from the patient was taken regarding the procedure prior to examination. Subjects were enrolled in a case control study within $24 \mathrm{hrs}$ of delivery in the hospital.The study consisted of two groups with 100 subjects each: Group I: (Control): Defined as mothers who delivered infants weighing more than or equal to $2500 \mathrm{~g}$ and with a gestational age of 37 weeks or more (Term normal weight infants, TNBW).Group II: (Case): This group was further categorized into: Subgroup A: (Preterm normal weight, PNBW): Defined as mothers who delivered infants weighing more than or equal to $2500 \mathrm{~g}$ and with a gestational age of less than 37 weeks. Subgroup B: (Term low birth weight, TLBW): Defined as mothers who delivered infants weighing less than $2500 \mathrm{~g}$ and with a gestational age of 37 weeks or more.Subgroup C: (Preterm low birth weight, PTLBW): Defined as mothers who delivered infants weighing less than $2500 \mathrm{~g}$ and with a gestational age of less than 37 weeks.

Information on current and historical pregnancy outcomes were obtained by taking detailed history verbally from the patient about the previous pregnancy outcomes and outcome of current pregnancy. Hospital record of the patient was verified for the obstetric risk factors, birth weight of the new born and the weight of the mother. Consent regarding the nutritional status and built of the patient were taken from the department of Medicine and Gynecology. The details about the habits like tobacco smoking, pan chewing, drug abuse was obtained from the patient.Inclusion criteria: Postpartum mothers were selected for the study within 24 hours after the delivery moderately to well-built patients were selected for the study. Patients who conceived naturally and delivered normally were taken into consideration. The subjects were in the age group of 18-35 years.Exclusion criteria: No history of any systemic diseases or systemic infections during pregnancy. No history of use of any medications such as corticosteroids or antibiotics during pregnancy. No history of tobacco or alcohol use. No history of previous preterm birth, low birth weight, preterm low birth weight infants, spontaneous abortions, still births and extremely high parity or gravidity. Body mass index not less than 19.8 $\mathrm{kg} / \mathrm{m}^{2}$ The gingival and periodontal status of both the groups was assessed within $24 \mathrm{hrs}$ of delivery by using the following indices: Gingival Index (Loe and Silness), Community periodontal index (CPI)

Statistical analysis: Descriptive data that included mean and standard deviations were determined for each of the variables in each group. Categorical data was analyzed by Chi-square test. Multiple groups were compared by one-way ANOVA and group-wise by Student's t-test. Relationship between gingival status and the community periodontal index score and the other variables was assessed by Pearson's correlation coefficient. Significant for all the tests was predetermined at a probability value of 0.05 or less.

\section{Results}

Table 1 shows the comparison of gingival status of the mother between the controls and the cases. Most of the subjects had mild gingivitis in the Control group, moderate gingivitis in Subgroup A cases and severe gingivitis in Subgroup B cases and Subgroup C cases. Statistically there was a significant difference between the controls and the cases when the gingival status was compared $(\mathrm{P}<0.05)$. Table 2 shows the comparison of the community periodontal index score between the cases and the controls. Maximum number of subjects in the Control group showed bleeding on probing, while the Subgroup A cases showed pocket measuring $4-5 \mathrm{~mm}$ and the Subgroup B cases and Subgroup C cases demonstrated a pocket depth of $>6 \mathrm{~mm}$. There was a statistically significant difference between the controls and cases when the community periodontal index score was compared $(\mathrm{P}<0.05)$. Table 3 shows the relationship of the maternal age to the gingival status between the controls and the cases. The mean age of mothers in Control group was $22.93 \pm 2.53$ yrs for mild gingivitis and $24.4 \pm 3.75 \mathrm{yrs}$ for moderate gingivitis. This group showed significant association of maternal age with gingival status $(\mathrm{P}<0.05)$. The mean maternal age of subjects in the Subgroup A cases was $20.00 \pm 0.00 \mathrm{yrs}$ for mild gingivitis, $21.25 \pm 0.96$ yrs for moderate gingivitis and $21.00 \pm 0.00$ yrs for severe gingivitis. Thus, a statistically significant association was seen $(\mathrm{P}<0.05)$. The mean maternal age of subjects in the Subgroup B cases was $21.00 \pm 0.00$ yrs for mild gingivitis, $22.38 \pm 2.24$ yrs for moderate gingivitis and $23.86 \pm 2.27$ yrs for severe gingivitis. The gingival status revealed severe gingivitis. A statistically significant association was found $(\mathrm{P}<0.05)$.The mean maternal age of subjects in the Subgroup C cases was $21.75 \pm 2.71$ yrs for mild gingivitis, $22.38 \pm 2.24$ yrs for moderate gingivitis and $23.62 \pm 1.98$ yrs for severe gingivitis. The gingival status of this group demonstrated severe gingivitis. Statistically a significant association was seen $(P<0.05)$.

Table 4, shows the relationship of the gestational age to the gingival status between the controls and the cases.The mothers with mild gingivitis in Control group delivered infants with mean gestational age of $38.31 \pm$ 0.97 weeks, days; and those with moderate gingivitis delivered infants with mean gestational age of $38.18 \pm$ 0.95 weeks, days. This group showed a significant association of gestational age with the gingival status $(\mathrm{P}<$ 0.05).The mothers with mild gingivitis in Subgroup A cases delivered infants with mean gestational age of $37.50 \pm 0.00$ weeks, days; those with moderate gingivitis delivered infants with mean gestational age of $38.70 \pm$ 1.37 weeks, days; and those with severe gingivitis delivered infants with mean gestational age of $37.40 \pm 0.00$ 
weeks, days. This group showed severe gingivitis. Statistically, a significant association was found. The mothers with mild gingivitis in Subgroup B cases delivered infants with mean gestational age of $36.20 \pm 0.00$ weeks, days; those with moderate gingivitis delivered infants with mean gestational age of $35.45 \pm 0.39$ weeks, days; and those with severe gingivitis delivered infants with mean gestational age of $35.34 \pm 0.14$ weeks, days. This group revealed severe gingivitis. There was statistically significant difference $(\mathrm{P}<0.05)$. The mothers with mild gingivitis in Subgroup B cases delivered infants with mean gestational age of $35.61 \pm 0.63$ weeks, days; those with moderate gingivitis delivered infants with mean gestational age of $34.94 \pm 2.10$ weeks, days; and those with severe gingivitis delivered infants with mean gestational age of $35.60 \pm 0.40$ weeks, days. This group also revealed severe gingivitis and showed a statistically significant association $(\mathrm{P}<0.05)$.

Table 5, shows the relationship of the weight of the infant to the gingival status between the controls and the cases.The mothers with mild gingivitis in Control group delivered infants weighing $2.93 \pm 0.24 \mathrm{~kg}$; and those with moderate gingivitis delivered infants weighing $2.87 \pm 0.20 \mathrm{~kg}$. Most of the mothers in this group had mild to moderate gingivitis. This group showed a significant association of weight of the infant with gingival status $(\mathrm{P}<0.05)$. The mothers with mild gingivitis in Subgroup A cases delivered infants weighing $2.23 \pm 0.00$ $\mathrm{kg}$; those with moderate gingivitis delivered infants weighing $2.23 \pm 0.10 \mathrm{~kg}$ and those with severe gingivitis delivered infants weighing $2.23 \pm 0.00 \mathrm{~kg}$. Most of the mothers in this group showed moderate to severe gingivitis. A significant association of weight of the infant with gingival status was found $(\mathrm{P}<0.05)$.The mothers with mild gingivitis in Subgroup B cases delivered infants weighing $2.50 \pm 0.00 \mathrm{~kg}$; those with moderate gingivitis delivered infants weighing $2.68 \pm 0.15 \mathrm{~kg}$; and those with severe gingivitis delivered infants weighing $2.53 \pm 0.05 \mathrm{~kg}$. The mothers in this group showed moderate to severe gingivitis. There was a statistically significant difference seen $(\mathrm{P}<0.05)$. The mothers with mild gingivitis in Subgroup $\mathrm{C}$ cases delivered infants weighing $2.14 \pm 0.23 \mathrm{~kg}$; those with moderate gingivitis delivered infants with mean infant weight of $2.09 \pm 0.22 \mathrm{~kg}$; and those with severe gingivitis delivered infants weighing $2.22 \pm 0.14 \mathrm{~kg}$. The mothers in this group showed moderate to severe gingivitis. There was a statistically significant difference seen $(\mathrm{P}<0.05)$.

Graph 1 shows the relationship of the maternal age to the community periodontal index score between the controls and the cases. The mean age of mothers in Control group who exhibited bleeding on probing was $23.09 \pm 2.75 \mathrm{yrs}$, and those who exhibited pockets measuring $4-5 \mathrm{~mm}$ was $22.00 \pm 0.00 \mathrm{yrs}$. Most of the mothers in this group revealed bleeding on probing and pockets measuring 4-5 $\mathrm{mm}$. This group showed significant association of maternal age with periodontal status $(\mathrm{P}<0.05)$. The mean maternal age of subjects in the Subgroup A cases who exhibited pockets measuring $4-5 \mathrm{~mm}$ was $21.00 \pm 1.00 \mathrm{yrs}$, and those who exhibited pockets measuring $>6 \mathrm{~mm}$ was $21.00 \pm 0.00$ yrs. This group demonstrated pockets measuring $>6 \mathrm{~mm}$. A statistically significant association was seen $(\mathrm{P}<0.05)$.The mean maternal age of most subjects in the Subgroup B cases who exhibited pockets measuring $4-5 \mathrm{~mm}$ was $21.00 \pm 1.41 \mathrm{yrs}$, and those who exhibited pockets measuring > $6 \mathrm{~mm}$ was $24.63 \pm 2.13$ yrs. The periodontal condition showed pockets measuring $>6 \mathrm{~mm}$. A statistically significant association was found $(\mathrm{P}<0.05)$ The mean maternal age of subjects in the Subgroup $\mathrm{C}$ cases who exhibited pockets measuring $4-5 \mathrm{~mm}$ was $22.30 \pm 2.52 \mathrm{yrs}$, and those who exhibited pockets measuring $>6 \mathrm{~mm}$ was $23.25 \pm 2.02 \mathrm{yrs}$. The periodontal status of this group demonstrated pockets measuring > $6 \mathrm{~mm}$. Statistically, a significant association was seen $(\mathrm{P}<0.05)$.

Graph 2 shows the relationship of the gestational age to the community periodontal index score between the controls and the cases.The mothers exhibiting bleeding on probing in the Control group delivered infants with mean gestational age of $38.30 \pm 0.97$ weeks, days; and those exhibiting pockets measuring 4-5 mm delivered infants with mean gestational age of $38.50 \pm 0.00$ weeks, days. Most of the mothers in this group revealed bleeding on probing and pockets measuring $4-5 \mathrm{~mm}$. This group showed significant association of maternal age with periodontal status $(\mathrm{P}<0.05)$. The mothers exhibiting pockets measuring $4-5 \mathrm{~mm}$ in Subgroup A cases delivered infants with mean gestational age of $38.46 \pm 1.30$ weeks, days; and those exhibiting pockets measuring $>6 \mathrm{~mm}$ delivered infants with mean gestational age of $37.40 \pm 0.00$ weeks, days. This group demonstrated pockets measuring $>6 \mathrm{~mm}$. A statistically significant association was seen $(\mathrm{P}<0.05)$. The mothers exhibiting pockets measuring 4-5 mm in Subgroup B cases delivered infants with mean gestational age of 35.68 \pm 0.51 weeks, days; and those exhibiting pockets measuring $>6 \mathrm{~mm}$ delivered infants with mean gestational age of $35.43 \pm 0.13$ weeks, days. This group demonstrated pockets measuring $>6 \mathrm{~mm}$. A statistically significant association was seen $(\mathrm{P}<0.05)$.The mothers exhibiting pockets measuring 4-5 $\mathrm{mm}$ in Subgroup $\mathrm{C}$ cases delivered infants with mean gestational age of $35.24 \pm 1.04$ weeks, days; and those exhibiting pockets measuring $>6 \mathrm{~mm}$ delivered infants with mean gestational age of $35.43 \pm 1.46$ weeks, days. The community periodontal index score revealed pockets measuring $>6 \mathrm{~mm}$. There was a statistically significant association $(\mathrm{P}<$ $0.05)$.

Graph 3 shows the relationship of the weight of the infant to the community periodontal index score between the controls and the cases.The mothers exhibiting bleeding on probing in the Control group delivered infants weighing $2.63 \pm 0.24 \mathrm{~kg}$; and those exhibiting pockets measuring $4-5 \mathrm{~mm}$ delivered infants weighing 
$3.20 \pm 0.00 \mathrm{~kg}$. Most of the mothers in this group revealed bleeding on probing and pockets measuring $4-5 \mathrm{~mm}$. This group showed significant association of maternal age with periodontal status $(\mathrm{P}<0.05)$. The mothers exhibiting pockets measuring 4-5 mm in Subgroup A cases delivered infants weighing $2.24 \pm 0.09 \mathrm{~kg}$; and those exhibiting pockets measuring $>6 \mathrm{~mm}$ delivered infants weighing $2.30 \pm 0.00 \mathrm{~kg}$. This group demonstrated pockets measuring $>6 \mathrm{~mm}$. A statistically significant association was seen $(\mathrm{P}<0.05)$. The mothers exhibiting pockets measuring 4-5 mm in Subgroup B cases delivered infants weighing $2.60 \pm 0.14 \mathrm{~kg}$; and those exhibiting pockets measuring $>6 \mathrm{~mm}$ delivered infants weighing $2.356 \pm 0.11 \mathrm{~kg}$. A statistically significant association was seen $(\mathrm{P}<0.05)$ The mothers exhibiting pockets measuring $4-5 \mathrm{~mm}$ in Subgroup $\mathrm{C}$ cases delivered infants weighing $2.08 \pm 0.25 \mathrm{~kg}$, and those exhibiting pockets measuring $>6 \mathrm{~mm}$ delivered infants weighing $2.21 \pm 0.14$ $\mathrm{kg}$. The community periodontal index score revealed pockets measuring $>6 \mathrm{~mm}$. There was a statistically significant association $(\mathrm{P}<0.05)$

Table 1: Comparison Of Gingival Status In Controls And Cases

\begin{tabular}{|l|c|c|c|c|c|}
\hline \multicolumn{1}{|c|}{$\begin{array}{c}\text { Gingival } \\
\text { Status }\end{array}$} & Controls & $\begin{array}{c}\text { Subgroup } \\
\text { A }\end{array}$ & Subgroup B & Subgroup C & Significance \\
\hline Mild & 90 & 1 & 1 & 8 & \multirow{2}{*}{$\begin{array}{c}2 \\
\text { P }\end{array}<0.207$} \\
\hline Moderate & 10 & 4 & 4 & 29 & \\
\hline Severe & 0 & 1 & 7 & 45 & \\
\hline
\end{tabular}

Table 2: Comparison Of Periodontal Status In Controls And Cases

\begin{tabular}{|l|c|c|c|c|c|}
\hline \multicolumn{1}{|c|}{ Periodontal Status } & Controls & Subgroup A & Subgroup B & Subgroup C & Significance \\
\hline Bleeding & 99 & 0 & 0 & 0 & $\chi^{2}=208.309$ \\
Pockets 4-5 mm & 1 & 5 & 4 & 27 & P $<05$ \\
\hline Pockets $\geq 6 \mathrm{~mm}$ & 0 & 1 & 8 & 55 & \\
\hline
\end{tabular}

Table 3: Relationship Of Maternal Age With Gingival Status In Controls And Cases

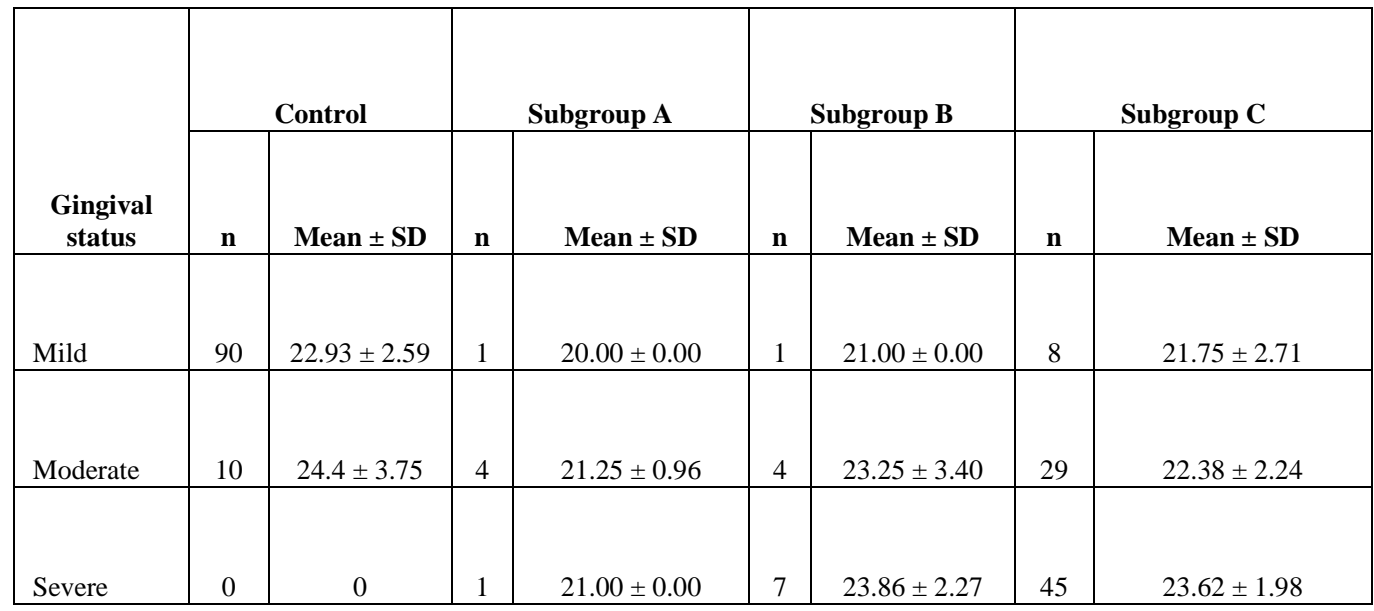

Table 4: Relationship Of Gestational Age With Gingival Status In Controls And Cases

\begin{tabular}{|l|c|c|c|c|c|c|c|c|}
\hline \multirow{2}{*}{$\begin{array}{c}\text { Gingival } \\
\text { status }\end{array}$} & \multicolumn{2}{|c|}{ Control } & \multicolumn{2}{c|}{ Subgroup A } & \multicolumn{2}{c|}{ Subgroup B } & \multicolumn{2}{c|}{ Subgroup C } \\
\cline { 2 - 8 } & $\mathbf{n}$ & Mean \pm SD & $\mathbf{n}$ & Mean \pm SD & n & Mean \pm SD & n & Mean \pm SD \\
\hline Mild & 90 & $38.31 \pm 0.97$ & 1 & $37.50 \pm 0.00$ & 1 & $36.20 \pm 0.00$ & 8 & $35.61 \pm 0.63$ \\
\hline Moderate & 10 & $38.18 \pm 0.95$ & 4 & $38.70 \pm 1.37$ & 4 & $35.45 \pm 0.39$ & 29 & $34.94 \pm 2.10$ \\
\hline Severe & 0 & 0 & 1 & $37.40 \pm 0.00$ & 7 & $35.34 \pm 0.14$ & 45 & $35.60 \pm 0.44$ \\
\hline
\end{tabular}

Table 5: Relationship Of Weight Of The Infant With Gingival Status In Controls And Cases

\begin{tabular}{|l|c|c|c|c|c|c|c|c|}
\hline \multirow{2}{*}{$\begin{array}{c}\text { Gingival } \\
\text { status }\end{array}$} & \multicolumn{2}{|c|}{ Control } & \multicolumn{2}{c|}{ Subgroup A } & \multicolumn{2}{c|}{ Subgroup B } & \multicolumn{2}{c|}{ Subgroup C } \\
\cline { 2 - 9 } & $\mathbf{n}$ & Mean \pm SD & $\mathbf{n}$ & Mean \pm SD & n & Mean \pm SD & n & Mean \pm SD \\
\hline Mild & 90 & $2.93 \pm 0.24$ & 1 & $2.30 \pm 0.00$ & 1 & $2.50 \pm 0.00$ & 8 & $2.14 \pm 0.23$ \\
\hline Moderate & 10 & $2.87 \pm 0.20$ & 4 & $2.23 \pm 0.10$ & 4 & $2.68 \pm 0.15$ & 29 & $2.09 \pm 0.22$ \\
\hline Severe & 0 & 0 & 1 & $2.30 \pm 0.00$ & 7 & $2.53 \pm 0.05$ & 45 & $2.22 \pm 0.14$ \\
\hline
\end{tabular}




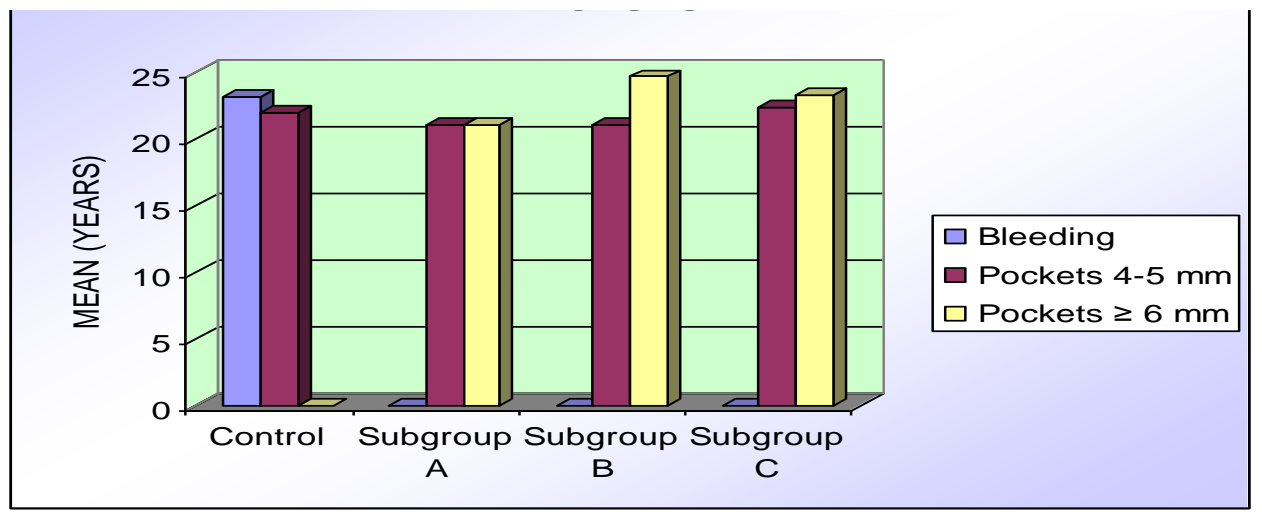

Graph 1- Comparison Of Maternal Age Of Infant With Periodontal Status In Controls And Cases

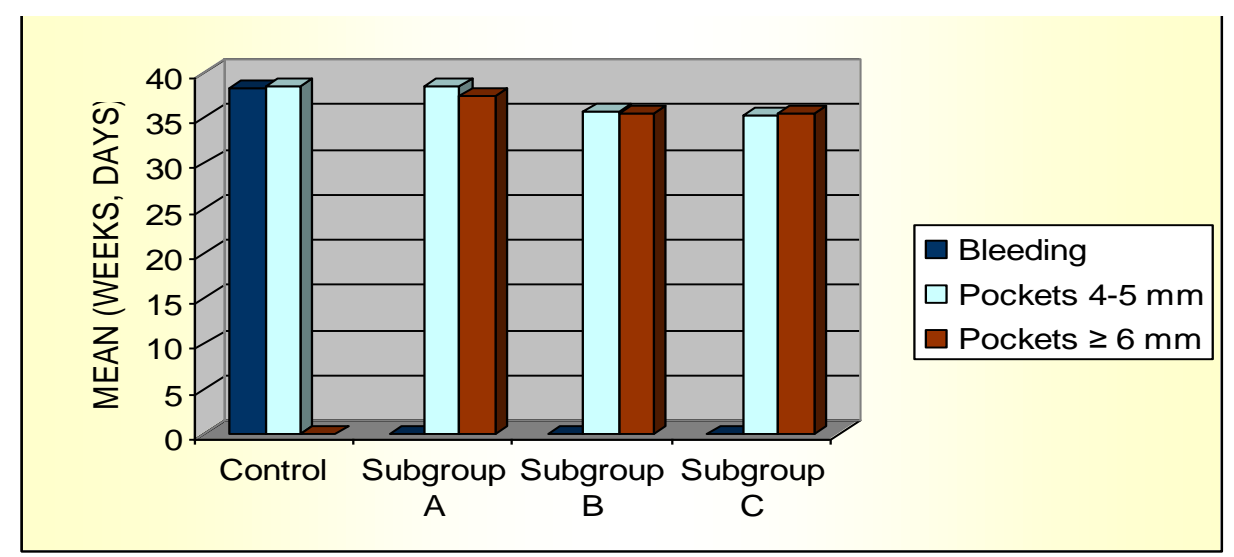

Graph 2- Comparison Of Gestational Age Of Infant With Periodontal Status In Controls And Cases

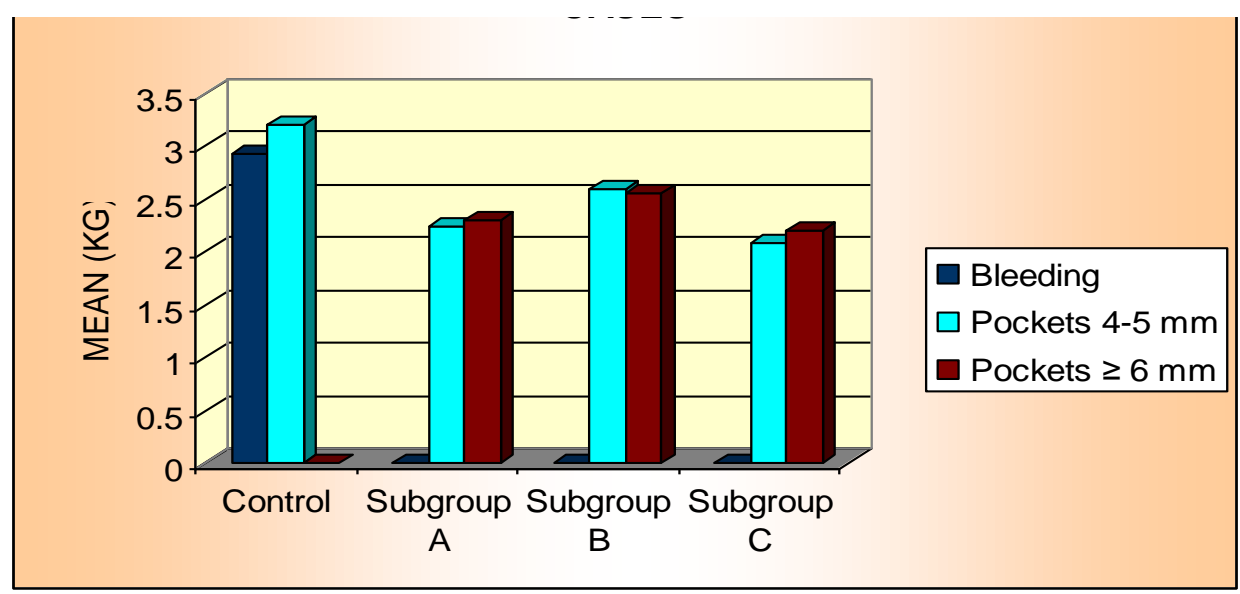

Graph 3- Comparison Of Weight Of Infant With Periodontal Status In Controls And Cases

\section{Discussion}

Preterm low birth weight is considered to be the foremost problem in obstetrical medicine and remains the leading cause of morbidity and mortality among neonates. Preterm low birth weight infants are at higher risk for a number of acute and chronic disorders, including respiratory distress syndrome, cerebral palsy; pathologic heart conditions, epilepsy, and severe learning problems. Most of the neonatal deaths are associated with 
preterm delivery and birth weight of under $1500 \mathrm{~g}$. Thus, birth weight is considered to be important determinant of the chances of an infant to survive, grow and mature[1].Multiple factors have been associated with the delivery of preterm and / or low birth weight infants[2] The evidence suggests an infectious etiology for a large percentage of cases for preterm birth. Genitourinary tract infections, such as bacterial vaginosis, and inflammatory mediators resulting from such infections have been considered a biologically plausible pathway for preterm labor and premature rupture of the membranes. Alternatively, it was hypothesized that preterm low birth weight may be indirectly mediated through distant infections resulting in translocation of bacterial vesicles and lipopolysaccharide (LPS) in the systemic circulation. However the exact mechanisms for the proposed relationship remain unclear.

Periodontal diseases are a group of infectious diseases resulting in inflammation of gingival and periodontal tissues and progressive loss of alveolar bone. The periodontal infection is initiated and sustained by several bacteria, predominantly gram-negative, anaerobic and microaerophilic bacteria that colonize the subgingival area. Host defense mechanisms play integral role in the pathogenesis of periodontal disease. It has been postulated that the association between periodontal disease and preterm / low birth weight (PLBW) may have similar pathogenic mechanisms as other maternal infections. Inflamed periodontal tissues produce significant amounts of pro-inflammatory cytokines, mainly Interleukin 1 (IL-1ß), IL-6, prostaglandin $\mathrm{E}_{2}$, and tumor necrosis factor alpha (TNF- $\alpha$ ), which may have systemic effects on the host. Hence, periodontal disease has the potential to influence preterm low birth weight through an indirect mechanism involving inflammatory mediators or a direct bacterial assault on the amnion. The study by Offenbacher and colleagues1996 [3]suggested that maternal periodontal disease could lead to a seven fold increased risk of delivery of a preterm tow birth weight infant.Collins and co-workers 1994 [5] reported that there was a 25\% reduction in birth weight in pregnant hamsters challenged subcutaneously in the dorsal region with the periodontal pathogen Porphyromonas gingivalis, compared with normal healthy pregnant hamsters. Human case-control studies have demonstrated that women who have low birth weight infants as a consequence of either preterm labor or premature rupture of membranes tend to have more severe periodontal disease than mothers with normal birth weight infants. When Gingival index was compared between Controls and Subgroup A Cases, Subgroup B Cases and Subgroup C Cases, there was a statistical significance. The above results agree with Samant et al 1976[7 ] they concluded that gingival changes are more marked than the periodontal changes seen during pregnancy. Hormonal changes due to estrogen and progesterone during pregnancy have a special effect on the periodontium, as vascular permeability increases in the gingival tissues, and, as a consequence, bacteria and / or their products can go through tissues more readily than normally.

When Community periodontal index score was between Controls and Subgroup A Cases, Subgroup B Cases and Subgroup C Cases, a statistically significant association was found. The above results agree with those reported by Mokeem SA, Molla GN, Al-Jewair TS 2004 [14]. They concluded that periodontal disease was more prevalent in mother's who delivered preterm low birth weight infants than in mothers who delivered term normal weight infants. This can be explained by the fact that periodontal disease may influence pregnancy outcome by the direct or the indirect effect of periodontal pathogens on the developing fetus.The relationship of maternal age with the gingival status was found to be significant in the Control group $(\mathrm{P}<0.05)$, Subgroup A Cases $(\mathrm{P}<0.05)$, Subgroup B Cases $(\mathrm{P}<0.05)$ and Subgroup $\mathrm{C}$ Cases $(\mathrm{P}<0.05)$. The above results agree with those reported by Dasanayake AP 1998[8]. He reported in his study that mothers who delivered pretem low birth weight infants appeared to have more severe gingivitis than the mothers who delivered term normal birth weight infants.

The relationship between the infant's birth weight and gingival status was significant in the Controls, Subgroup A Cases, Subgroup B cases and Subgroup C Cases. The above results are consistent with the findings of Dasanayake AP 1998.[8] This study reported that mothers with low birth weight had severe gingivitis when compared to mothers who delivered term normal birth weight infants. The relationship of the weight of the infant with the gingival status was found to be significant in the Control group $(\mathrm{P}<0.05)$, Subgroup A Cases $(\mathrm{P}<$ 0.05), Subgroup B Cases $(P<0.05)$ and Subgroup C Cases $(P<0.05)$. These results are concurrent with those of Marin et al [11] they concluded that bleeding on probing was significantly greater in women with infants weighing $<2500 \mathrm{~g}$ compared with women with infants weighing $2500-3499 \mathrm{~g}$ and $\geq 3500 \mathrm{~g}$. The relationship of maternal age with the periodontal condition was found to be significant in the Control group ( $\mathrm{P}<0.05)$, Subgroup A Cases $(\mathrm{P}<0.05)$, Subgroup B Cases $(\mathrm{P}<0.05)$ and Subgroup C Cases $(\mathrm{P}<0.05)$. The above results agree with those reported by Offenbacher $\mathrm{S}$ et al 1996 [7] They concluded that preterm low birth weight cases had worse periodontal disease than the respective normal birth weight controls. A study by Radnai M et al 2004 [12] indicated that early localized periodontitis of the patient during pregnancy can be regarded as an important risk factor for Preterm birth.The relationship between the infant's gestational age and periodontal condition was significant in the Control group, Subgroup A Cases and Subgroup B Cases and Subgroup C cases. The above results are concurrent with those reported by Jeffcoat MK et al 2001 [9] They concluded that there is an 
association between the presence of periodontitis at 21 to 24 weeks of gestation and subsequent preterm birth.A study by López NJ et al 2002[10] primarily assessed the delivery at less than 37 weeks of gestation or an infant weighing less than $2,500 \mathrm{~g}$ and found that periodontal disease appears to be an independent risk factor for PLBW.

The relationship between the weight of the infant and periodontal condition was found to be significant in the Control group, Subgroup A Cases, Subgroup B Cases and Subgroup C cases. The results are in agreement with those of Romero et al 2002 [13] A decrease in the average newborn's weight and gestational age was observed as the level of periodontal disease increased and the correlation analysis demonstrated a highly significant clinical relationship between more severe periodontal disease and lower birth weight in their study. Collins JG et al 1994 [4] suggested that infection with Gram-negative periodontal pathogens may be due to adverse effects on the fetus, depending on the degree of infection. The authors reported that the lipopolysaccharide from oral bacteria can cause adverse pregnancy outcomes and have shown that increasing doses of lipopolysaccharide from both Escherichia coli and Porphyromonas gingivalis produced biphasic effects on fetal weight with significant decreases in fetal weight al higher doses. Dasanayake AP 1998[8] stated that poor periodontal health of the mother is a potential independent risk factor for low birth weight (LBW). The author suggested that periodontal disease, which is a Gram-negative anaerobic infection, can affect pregnancy outcome either by the direct or indirect effect of periodontal pathogens on the developing fetus.

In contrast, the study by Davenport et al 2002 [2] found no evidence that maternal periodontal disease is associated with preterm low birth weight. Indeed, they found evidence that increasing mean pocket depth at the time of delivery was associated with a reduction in risk for preterm low birth weight. The failure to control adequately for potential confounding factors may be one reason for inconsistencies between the results of various studies.Periodontal disease, which is a Gram-negative anaerobic infection, can affect pregnancy outcome either by the direct or indirect effect of periodontal pathogens on the developing fetus. While there is no evidence to date as to whether specific periodontal pathogens are found in relation to the developing fetus or the feto-placental unit, there is ample evidence for the effect of other infectious agents found in the genitourinary tract on the pregnancy outcome. Bacterial vaginosis and a high prevalence of maternal lower genitourinary tract infections are associated with poor pregnancy outcomes. Colonization or the vagina and cervix with Gram-negative Bacteroides is also associated with poor pregnancy outcomes. The possibility that the inflammation of the placental membranes could occur even without signs of infection, and that such inflammation is associated with poor pregnancy outcomes, lends credibility to the idea of an indirect effect of periodontal pathogens on the developing fetus. Offenbacher et al 1996 [7] hypothesized that Gram-negative anaerobic pathogens from the periodontium and associated endotoxins and maternal inflammatory mediators could have a possible adverse effect on the developing fetus. This view is further supported by the results obtained from animal models, where subcutaneous infection with a periodontal pathogen and experimental periodontal disease in pregnant hamsters resulted in decreased fetal growth as well as increase inflammatory mediator levels.

Periodontal infections can serve as a chronic reservoir of lipoligosaccharide, which could target the placental membranes via the blood stream, Lipoligosaccharide has been shown to elicit $1 \mathrm{~L}-1$ and PGE, production by the chorioamnionic and trophoblastie cells, a process often associated with preterm parturition. Alternately, inflammatory mediators such as $\mathrm{PGE}_{2}$ and TNF- $\alpha$ may be produced locally within the periodontium and, due to the potential high vascularity of this organ, act as a potential systemic source of feto-toxic cytokines. Furthermore, increased serum TNF- $\alpha$ levels, have recently been found to be associated with the extent of disease progression in periodontitis patients who are undergoing active attachment loss. [8]

It is also possible that there is an unknown genetic or environmental confounder, that is, an underlying condition that places a patient at risk for both periodontal disease and pre term low birth weight. Also, there could be an underlying hyper- responsive inflammatory trait that may place an individual at risk for both more severe periodontitis and pre term low birth weight. But the possibility that the presence of periodontal infection rendering the patient more susceptible to sub clinical bacterial vaginosis can be a factor for pre term low birth weight. Hence, further detailed investigation is needed. Also, considering the fact that the periodontal infection are both preventable and readily treated, these findings provide new opportunities for early intervention strategies to reduce the incidence of pre term low birth weight.

\section{Conclusion}

This study was conducted in two hundred Indian postpartum mothers at Fr. Muller's Hospital, Mangalore. From the results obtained the following conclusions were arrived at:

1.The severity of gingivitis was found to be increased in mothers who delivered term low birth weight infants, mothers who delivered pre term normal weight infants and mothers who delivered pre term low birth weight infantsas compared to mothers who delivered infants with normal birth weight.

2.The periodontal condition revealed pockets measuring $\pm 6 \mathrm{~mm}$ in mothers who delivered term low birth weight 
infants, mothers who delivered pre term normal weight infants and mothers who delivered pre term low birth weight infants as compared to mothers who delivered infants with normal birth weight and gestational age who showed bleeding on probing.

As evident from this study it can be deduced that a significant difference exist between good and poor gingival and periodontal status of subjects and the incidence of term low birth weight, pre term normal weight infants and preterm low birth weight infants. The data from the study provides evidence that periodontal disease in pregnant women represent a previously unrecognized and clinically important obstetric risk factor for preterm low birth weight.

\section{Refrences}

[1]. Mitchell-Lewis D, Engebretson SP, Chen J. Periodontal infections and pre-term birth: early findings from a cohort of young minority women in New York. Eur J Oral Sci 2001; 109(1):34-39.

[2]. Davenport ES, William CE, Sterene JA. Maternal periodontal disease and preterm low birth weight. J Dent Res 2002; 81(5): 313 318.

[3]. Offenbacher S, Katz V, Fertik G. Periodontal infection as a possible risk factor for preterm low birth weight. J Periodontol. 1996; 67(10): 1103-1113.

[4]. Collins JG. Effects of Escherichia coli and Porphyromonas gingivalis lipopolysaccharide on pregnancy outcome in the golden hamster. Infect. Immun 1994; 62: 4652 - 4655.

[5]. Belkys C. Romero et al. Relationship between Periodontal Disease in Pregnant Women and the nutritional condition of their Newborns. J Periodontol 2002; 73:1177-1183.

[6]. 6.Buduneli N, Baylas H, Buduneli E, Turkoglu O, Dahlen G: Evaluation of the relationship between smoking during pregnancy and subgingival microbiota. J Clin Periodontol 2005; 32: 68-74.

[7]. Samant A., Malik. Gingivitis and periodontal disease in pregnancy. J Periodontol 1976; 47: 415 -418.

[8]. Dasanayake AP. Poor periodontal health of the pregnant woman as a risk factor for low birth weight. Ann Periodontol 1998; 3(1):206-12.

[9]. Jeffcoat MK, Geurs NC, Reddy MS, Liver SP, Goldenberg RL, Hauth JC. Periodontal infection and preterm birth. Results of a prospective study. J Am Dent Assoc 2001; 132:875-880.

[10]. López NJ. Periodontal Therapy May Reduce the Risk of Preterm Low Birth Weight in Women with Periodontal Disease: A randomized Controlled Trial. J Periodontol 2002; 73:911-924.

[11]. Marin C: Correlation between infant birth weight and mother's periodontal status. J Clin Periodontol 2005; 32: $299-304$.

[12]. Radnai M. A possible association between preterm birth and early periodontitis. Pilot study. J Clin Periodontol 2004; 31: 736-741

[13]. Belkys C. Romero.Relationship between Periodontal Disease in Pregnant Women and the nutritional condition of their Newborns. J Periodontol 2002; 73:1177-1183.

[14]. Mokeem SA, Molla GN, Al-Jewair TS. The Prevalence and Relationship between Periodontal Disease and Pre-term Low Birth Weight Infants at King Khalid University Hospital in Riyadh, Saudi Arabia. J Contemp Dent Pract 2004 ;( 5)2:040-056. 